\title{
EFFECTS OF NON IONIZED ELECTROMAGNETIC RADIATION ON SATELLITES
}

\author{
Ajay Pathania', Vijay Dadhich ${ }^{2}$ \\ ${ }^{1}$ B.Tech Aerospace+ M.Tech Avionics, Aerospace Department, Amity University, UP, India \\ ${ }^{2}$ M.Tech Automobile Engineering, Galgotias University, Utter Pradesh, India
}

\begin{abstract}
This paper discuss about the Electromagnetic radiation Interference in the satellites. It covers maximum avionics systems and the affects of non ionized electromagnetic radiations on various components. This paper also tells us about the various important factors that should be always in the mind while start designing any satellite.

It is a case study of various satellites as Chandrayaan, Resourcesat and many more. This study is made to analyze and ensure that the best technique can be opted for the Electromagnetic interference and compatibility. It is clear that the electromagnetic compatibility of the satellites is always differing from each other as depend on the payload which is carried by it. But there are some common EMI techniques which can be applied in general and we can notice the general effects of Non Ionized EM radiations on the avionics portion of the satellites.
\end{abstract}

Keywords: EM radiations, electromagnetic radiations, electromagnetic radiations on satellites, EMI-EMC, satellite design...

\section{INTRODUCTION}

Establishing EMC in a complex spacecraft is a challenging job due to the presence of varied, complex and more sensitive systems, RF near fields and densely packed electronic systems operating in various frequency ranges. So that the interference due to ionized radiations as well as due to non- ionized radiation become very common.

The case study is done with the various satellites as Resourcesat2, Chandrayaan-1, GSAT-11, GSAT-8 and INSAT-3D. The general info about these satellites is given below which is taken from the internet sources.

\subsection{Resourcesat-2}

This spacecraft is configured with improved features like $70 \mathrm{Km} \mathrm{Mx}$ Data, enhanced SSR memory of $400 \mathrm{~GB}$, improved Radiometric accuracy from 7 bits to 10 bits for LISS- 3 and LISS- 4 and 10 bits to 12 bits for AWIFS, new Data Handling system, 10/8 channel satellite Positioning System (SPS), Star Sensor, AOCE with MIL-1553 and miniaturized Payload electronics. It was launched on April 20th, 2011 from Satish Dhawan Space Centre in the PSLVC16 carrier rocket [9].

\subsection{Chandrayaan-1}

Chandrayaan-1, India's first mission to Moon, was launched successfully on October 22, 2008 from SDSC SHAR, Sriharikota. The spacecraft was orbiting around the Moon at a height of $100 \mathrm{~km}$ from the lunar surface for chemical, mineralogical and photo-geologic mapping of the Moon. The spacecraft carries 11 scientific instruments built in
India, USA, UK, Germany, Sweden and Bulgaria. Till the time this report was written Chandrayaan had achieved many mile stone as searching water on the moon was the biggest one [10].

\subsection{GSAT-11}

GSAT-11 will be developed at the cost of Rs. 500 crore. At 4.5 tones, it will weigh more than twice as much as biggest Indian satellite in orbit now. With 16 high capacity multibeams in $\mathrm{Ku} / \mathrm{Ka}$ band, GSAT-11 will provide much faster uplinks for a host of communications and broadcasting services, including direct-to-home (DTH television). With a dry mass of 2.1 tones, the spacecraft will provide $10 \mathrm{GHz}$ of bandwidth, which will be equivalent to about 220 transponders of $36 \mathrm{MHz}$ The advanced satellite, will employ a new 1-4K Bus (computer network). It will be configured with two-sided large solar array panels generating $11 \mathrm{KW}$ of power.

After the successful completion of all the major mission objectives, the orbit has been raised to $200 \mathrm{~km}$ during May 2009 [5].

\subsection{GSAT-8}

GSAT-8, India's advanced communication satellite, is a high power communication satellite being inducted in the INSAT system. Weighing about $3100 \mathrm{Kg}$ at lift-off, GSAT8 is configured to carry 24 high power transponders in $\mathrm{Ku}-$ band and a two-channel GPS Aided Geo Augmented Navigation (GAGAN) payload operating in L1 and L5 bands. The $24 \mathrm{Ku}$ band transponders will augment the 
capacity in the INSAT system. The GAGAN payload provides the Satellite Based Augmentation System (SBAS), through which the accuracy of the positioning information obtained from the GPS Satellite is improved by a network of ground based receivers and made available to the users in the country through the geostationary satellites [4].

\subsection{INSAT-3D}

INSAT-3D is a meteorological, data relay and satellite aided search and rescue satellite developed by the Indian Space Research Organization and expected to be launched in 2011 using an ARIANESPACE launch vehicle. The configuration of INST-3D consists of a state of art Meteorological Payload as the main payload. The configuration of the spacecraft is to a large extent constrained by the launcher (GSLV) compatibility requirements. It is to be compatible with first operational flight of GSLV with mass of $2000 \mathrm{~kg}$. The dynamic envelope available with GSLV is $3050 \mathrm{~mm}$.

Till now we have discussed about the various satellites. These all satellites are used as case studies various factors of these which were considered to be common were taken and analyzed. Finally we get the information that the design is the key factor in the satellite. If we will take care of EMI EMC related problems from the beginning of the design then we will be able to make a system which will be compatible with the high and low frequency components [1].

\section{RADIATED EMISSIONS FROM SPACECRAFT}

It is not only the non ionized electromagnetic radiation or conducting emission but also The Radiated Emissions correspond to RF signals radiated from high power equipments (transmitters) which can affect the performance of low power equipments (receivers). The radiated emissions correspond to the RF signals radiated from high power equipments, transmitters, which can affect the performance of low power equipments, receivers. The maximum electric field levels generated by the spacecraft and payload elements, by virtue of transmitting antennas, are determined in order to confirm that the equipment radiated susceptibility requirements specified have sufficient margins. Radiated Emissions and Radiated Susceptibility analysis have been carried out for Resourcesat-2 spacecraft. The isolation and shielding values used in these analyses are estimated from the past performance of the each equipment type used and requirements specified in the appropriate equipment specifications. The level of Electric field radiated from the transmitting antenna reaching sensitive equipments is calculated using the model given below. The calculation is carried out for both transmit and receive frequency bands [2], [3].

\section{HOW THINGS HAPPEN AND WHY???}

\subsection{EDS}

EDS stands for Electrical Distribution System. EDS that provides the connectivity for the interfaces of various subsystems, is the major path of electromagnetic coupling. For power distribution, twisted shielded pair wires are used. Twisting of wires reduces the residual magnetic field within the spacecraft which is essential for error free operation of magnetic torquers. Additional shielding measures are ensured for cables connected to Electro Explosive Devices (EED) and low-level signal carrying cables.

Single core shielded wires used for low level analog signals such as preamplifier outputs of sensors. Shield of coaxial cables are terminated on structural ground for better shielding effectiveness. Pigtail lengths were minimized. Unshielded wires are employed only for low frequency signals while Semi rigid coaxial cables are used for RF and flexible coaxial cables high frequency clock signals [11].

Data transfer between payload elements and BDH packages and between $\mathrm{BDH}$ and Solid State Recorders is in differential mode through LVDS cable, as the differential data transfer is less susceptible to common mode noise. Pins allocation on a connector are done in such a way that the low level signals pins are surrounded by ground pins to avoid any coupling.

\subsection{Spacecraft Layout Design}

Spacecraft layout is the most important factor of the design. Spacecraft Layout design involves evolving a viable scheme for placement of different subsystem components meeting all the requirements. The layout identifying the locations of the different packages is worked out to meet the following requirements:

- Placement of subsystems, based on the EMC.

- Separate harness corridor for signal and power.

Subsystems placement on spacecraft layout is constrained by factors like interface and functional requirement, in addition to Non Ionized electromagnetic considerations. Subsystems having signal flow among them are kept close to each other.

If there is a flaw in the layout small interference from one component can cause a big disaster in the satellite [14].

\subsection{Spacecraft Grounding Scheme}

The grounding scheme can be split into two categories: Subsystem level grounding scheme and system level grounding scheme. The Subsystem level grounding scheme specifies that the analog and digital ground called as secondary grounds are brought as a single sub-star point which are referred to the panel reference ground through $12 \mathrm{AWG}$ wires. The relay driver ground is connected back to power returns to avoid any current flow in ground reference during relay operations. The primary bus returns are connected to bus returns in power subsystem and are not 
mixed-up with secondary returns of the subsystems [6], [7].

\subsection{Distributed Single Point Grounding Scheme}

The principle of DSPG is to realize a single point grounding of each independent power network and galvanic isolation between these networks. DSPG is one of the secondary / signal grounds referencing scheme. In DSPG, each assembly has one and only one path to the chassis and there are no deliberate structure currents.

To implement the DSPG in the satellites following factors can be taken into consideration:

Primary power return from Power package should be connected to SRS from a single point.

Secondary power shall be grounded to structure only once in each unit. To avoid common mode effects the S/C structure shall not be used as return path for power and signals.

Electrical interfaces between sub-systems should be differential in nature [7].

\subsection{Structural Design}

The spacecraft structure has the following major roles from EMI/EMC/ESD aspects. Here we are considering and comparing EMI EMC or ESD with the Non Ionized electromagnetic radiation:

- To ensure a good shielding efficiency by being a Faraday cage in order to protect the inner electronics from the outer electromagnetic fields generated by the payload or by possible electrostatic discharges.

- $\quad$ To ensure a good protection against bulk charging. Therefore the continuity between all panels is the object of primary importance.

- To ensure a good electrical ground reference plane in order to provide an equipotential reference for the electronics. The common mode voltage between any two points of the structure shall not exceed $50 \mathrm{mV}$, under normal conditions [12].

\section{ESD IN SATELLITES}

This is important to minimize the ESD occurrence risks. This is done by the application of design rules detailed in the subsequent section and by numerical analysis which predicts the static potential on the external material parts of the satellite (NASCAP analysis) which allows verifying that dangerous values are not reached [15].

\subsection{ESD Design}

Grounding all conductive elements to a common ground will minimize potential differences. Similarly, making all spacecraft exterior surfaces at least partially conductive minimizes differences as well. The main ESD control guidelines are given below, which are applicable both at the subsystem and system levels:

1. The primary spacecraft structure, electronic components housings and electric cable shields provide a physically and electrically continuous shielded surface around all electronics and wiring.

2. The conductive material on antenna reflector rear surface should be grounded to the structure.

3. Wire shields should not be used as return lines except in case of co-axial cables.

4. Twisted and shielded pair wires should be used, except on the solar panels.

5. The electrical continuity of the spacecraft grounding system should intact with less than $2.5 \mathrm{~m} \Omega$ / joint.

\subsection{ESD Control Measures}

A spacecraft in a geo-synchronous orbit is likely to be charged by the plasma environment to high potentials. This builds up of charge on external surfaces of a spacecraft is referred to as spacecraft charging. Spacecraft charging can be classified into two types. The first, called the absolute charging, occurs when the entire spacecraft is charged negatively with respect to ambient plasma environment. The second type called the differential charging occurs when parts of the spacecraft are charged to different negative potentials relative to each other. Absolute charging occurs very rapidly during magnetic sub-storms and in a fraction of second during eclipse. Differential charging usually occurs slowly and results in one part of surface being charged to a potential different from other parts of the spacecraft.

\section{HARNESS DESIGN}

The harness is designed to minimize cross talk, radiation and noise pick up. Cable routing will be as close as possible to the ground reference plane. No cables are routed on the outside surfaces of the satellite, except when unavoidable.

To ensure a better immunity of the harness with regard to a coupling with an electric / magnetic field or a coupling with any other harness, the shield shall be grounded to the structure at both ends.

At connector interface, terminations of shields are designed to get a minimum of unshielded wire portion. Wires of any one group may be assembled together in bundles but wires of different groups shall be kept separated as far as possible [13].

\section{CALCULATION AND OBSERVATIONS}

The maximum RF power within equipment radiates isotropically and the E-field value at a distance of $r$ meter is computed using the following power flux density equation.

$$
\mathrm{E}^{2} /(120 \pi)=\mathrm{PG} / 4 \pi \mathrm{r} 2 \text { watts } / \mathrm{m}^{2}
$$

Where, $\mathrm{E}$ is the electric field in Volts/m

$\mathrm{P}$ is the maximum $\mathrm{RF}$ power in $\mathrm{W}$

$r$ is the distance in $m$

$\mathrm{G}$ is the transmitting source gain $(\mathrm{G}=1$, isotropic Radiator)

\section{EMI CONTROL MEASURES}

It is necessary to adopt EMI control measures from initial phase as well as various stages of spacecraft/satellite realization to ensure reliable performance of its payloads 
and all main frame elements.

\subsection{Filtering}

- Sharp roll-off filters should be used correctly to avoid any inter-channel interference.

- Specially designed fourth order filters for each type of DC/DC converters should be included in power line.

- Decoupling capacitors can also be used to reduce the transmission of board generated noise and prevent the transmission of digital switching noise.

- For improved regulation of DC supply, 3T regulators should be used.

\subsection{Shielding}

Electronic circuitry is housed in metallic boxes to provide necessary RF shielding. The package housing meets the following requirements:

- Good electrical contact with spacecraft structure using fasteners ensures the less bonding resistance.

- Provisions of cut-outs in housing for mounting electrical connectors without letting the RF shielding to suffer.

- Shielding should be done in that manner which will ensure minimum bonding resistance between different modules of package housing [12], [13].

\subsection{PCB Layout}

At higher frequencies, PCB tracks act as transmission lines whose voltages and current varies according to the terminating impedance. In multilayer PCB boards design, following guidelines are followed:

- $\quad$ Signals lines and their returns should be very close to each other to minimize signal loop areas.

- $\quad$ Provisions made for decoupling capacitors located very close to active circuitry.

- $\quad$ Sensitive electronics packages like Data Handling System (BDH), Bus management Unit (BMU) incorporate mother board daughter board philosophy with multilayer PCB designs.

- Input signal lines, output signals lines, analog signal lines and digital signal lines and their returns are isolated.

\section{CONCLUSIONS}

The performances of the payloads during all testing phases will found to be satisfactory. It has been ensured that the integrated spacecraft does not generate electromagnetic interference that could adversely affect intra system and inter system electromagnetic performance. Thus finally we can conclude that the satellites designed by taking care of above these steps and techniques can be more better and compatible as compare to the previous one. We have learnt from the previous mistakes and try to recover them. We have also suggested some of the new techniques which will be also help in designing the compatible satellite.

\section{ACKNOWLEDGEMENTS}

We would like to give special thanks to VP Sandlash (Ret. Director, Defence Electronics Applications Laboratory (DEAL), Mentor, ITM University, Gurgaon) and also to AMSSS Saroun for the full support and all kinds of help we needed to complete this research.

\section{REFERENCES}

[1]. INSAT-3D Comprehensive Design Review, Doc. No. ISRO - ISAC - INSAT-3D - RR - 0880

[2]. MIL-STD-1541A: "Electromagnetic Compatibility Requirements for Space Systems", (Dec. 1987).

[3]. PSLV launch: PSLV C 16 a workhorse launch vehicle for India, http://economictimes.indiatimes.com/articleshow/8035732.c $\mathrm{ms}$

[4]. GSAT-8 communication satellite launched successfully, India's advanced communication satellite". Indian Space Research Organization, Retrieved 2013-03-13

[5]. 'Geo-Stationary Satellite', http://www.isro.org/satellites/gsat-8.aspx.

[6]. "Satellite",

Wikipedia

http://en.wikipedia.org/wiki/Satellite

[7]. How Satellites Work

http://www.howstuffworks.com/satellite.htm

[8]. GSAT 10 http://www.isro.org/satellites/gsat-10.aspx

[9].

http://www.isro.org/satellites/resourcesat-2.aspx

RESOURCESAT-2

[10].

Chandrayaan-1

http://www.isro.org/satellites/chandrayaan-1.aspx

[11]. NASA Satellite Completes EMI/EMC Testing http://www.interferencetechnology.com/nasa-satellite-

completes-emiemc-testing/

[12]. NATIONAL RADIO ASTRONOMY

OBSERVATORY

http://apps.fcc.gov/ecfs/document/view;jsessionid=RGXQP

Hvc3NnctPrvhLqqSQ17JQyhpyXNyJmgw10Fnh7Z5V5T4

WBK!-1969853125!-1221852939?id=7021920701

[13]. Safer Cadmium Replacement for Military-Aerospace Connectors

http://apps.fcc.gov/ecfs/document/view;jsessionid=RGXQP Hvc3NnctPrvhLqqSQ17JQyhpyXNyJmgw10Fnh7Z5V5T4

WBK!-1969853125!-1221852939?id=7021920701

[14]. Satellite

http://ksp.kari.re.kr/html/korean/02_sub/sub02_02.html

[15]. Test Services

http://ksp.kari.re.kr/download/Satellite\%20AIT/Test\%20Ser

vices-Thermal\%20Environment\%20Test.pdf

\section{BIOGRAPHIES}

Ajay Pathania, the author is a prominent academic who has completed B.Tech Aerospace and M.Tech Avionics from Amity University, Noida-201301. He is astute, diligent and competent professional, with hands on experience on Research, Design, Development, Prototyping, Reverse Engineering, Testing, CAD/CAM/CAE and dealing with FEA/FEM, ANSYS, CFX, CFD, and CATIA for design, development and analysis of aeronautical products. 
Vijay Dadhich, The author is a prominent academic who has completed B. Tech Mechanical and M.Tech Automobile

Engineering, Galgotias University, Utter Pradesh, India. 